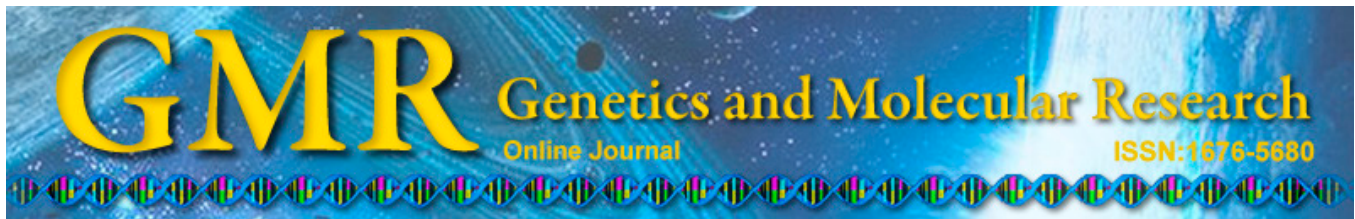

\title{
N-ethylmaleimide-sensitive factor siRNA improves cardiac function following myocardial infarction in rats
}

\author{
Y. Zhou ${ }^{1}$, Y. Liu ${ }^{2}$, S.X. Yang ${ }^{3}$ and Z. Wang ${ }^{1}$ \\ ${ }^{1}$ Emergency Department, \\ Peking University Ninth School of Clinical Medicine, Beijing, China \\ ${ }^{2}$ Emergency Department, The 302 Hospital of PLA, Beijing, China \\ ${ }^{3}$ Department of Cardiology, \\ Peking University Ninth School of Clinical Medicine, Beijing, China \\ Corresponding author: S.X. Yang \\ E-mail: yangsuixiang_1@163.com
}

Genet. Mol. Res. 14 (3): 9478-9485 (2015)

Received November 12, 2014

Accepted April 6, 2015

Published August 14, 2015

DOI http://dx.doi.org/10.4238/2015.August.14.11

ABSTRACT. This study examined the effects of N-ethylmaleimide-
sensitive factor (NSF) small interfering RNA (siRNA) on cardiac
function following myocardial infarction (MI) in rats. Thirty-six adult
Sprague Dawley rats were randomly divided into three equivalent groups.
An acute MI model was established by ligating the anterior descending
branch of the left coronary artery and confirmed by electrocardiogram.
Recombinant NSF-siRNA adenovirus (experimental), negative
adenovirus (control), and normal saline were injected near the infarcted
area of the left ventricle in each respective group. The left ventricular
ejection fraction (LVEF) was measured with a noninvasive ultrasonic
cardiogram. Left ventricular end-diastolic pressure (LVEDP) and the
maximum rate of rise in left ventricular pressure (+dp/dt max) were
measured using the BL-420 Biological Functional Experimental
System. Hearts were sectioned and stained with 2,3,5,-triphenyl
tetrazolium chloride (TTC) to observe the MI area. Two weeks after 
surgery, LVEF in the experimental group $(46.0 \pm 7.5 \%)$ was higher than control $(34.0 \pm 6.0 \%)$ and saline $(37.5 \pm 4.5 \%)$ group LVEFs $(\mathrm{P}<0.05)$, whereas LVEDP was the lowest in the experimental group (18.51 \pm 6.87 vs $29.47 \pm 9.94$ and $26.58 \pm 8.97 \mathrm{mmHg}$, respectively) $(\mathrm{P}<0.05)$. The $+\mathrm{dp} / \mathrm{dt}$ max was also higher in the experimental group $(9.74 \pm 1.16$ $v s 4.33 \pm 1.19$ and $5.24 \pm 1.53 \mathrm{mmHg} / \mathrm{s} \mathrm{x} \mathrm{10} 0^{3}$, respectively $)(\mathrm{P}<0.05)$; however, the MI area did not differ significantly between groups. Local injection of an adenovirus-mediated NSF-siRNA expression vector near infarcted areas improved cardiac function two weeks after MI, but had no impact on the MI area.

Key words: N-ethylmaleimide-sensitive factor; RNA interference; Cardiac function; Myocardial infarction

\section{INTRODUCTION}

N-ethylmaleimide-sensitive factor (NSF) is an important protein in endothelial cells that might mediate the merging of Weibel Palade bodies (WPBs) with the endothelial cell membrane. This juncture results in exocytosis and the release of WPB contents, primarily consisting of vasoactive substances such as von Willebrand factor, P-selectin, endothelin-1, and interleukin- 8 . These substances are released in the early stage of vascular injury. During this stage, neutrophils adhere to the blood vessel endothelium, and platelet aggregation occurs along with a series of inflammatory responses on the vascular wall such as vasospasms. These factors are closely related to the occurrence of atherosclerosis and of acute coronary syndrome (Lowenstein et al., 2005; Lowenstein and Tsuda, 2006). Our research group has studied the mechanism and regulation of WPB release (Yang et al., 2004), and we have successfully cloned the $N S F$ gene of human aortic endothelial cells and constructed a recombinant adenovirus vector containing an NSF small interfering RNA (siRNA) that targets the region of the gene encoding the NSF protein N-terminal functional domain in human aortic endothelial cells. After DNA sequencing and viral titering, we transfected the recombinant adenovirus $N S F$-siRNA vector into human aortic endothelial cells. Expression of NSF mRNA and protein was measured by real-time polymerase chain reaction and western blot technology at different time points. We found that $N S F$-siRNA induced a reduction in $N S F$ mRNA expression and decreased NSF protein synthesis, thus restraining the release of WPBs.

Based on previous studies, we established a myocardial infarction (MI) model in rats, and injected the recombinant adenovirus $N S F$-siRNA vector at multiple sites around the area of MI. We then observed the cardiac function and MI area after two weeks to evaluate the effects of $N S F$-siRNA on hearts following MI.

\section{MATERIAL AND METHODS}

\section{Material}

We purchased 36 specific-pathogen-free Sprague-Dawley male rats weighing 180$220 \mathrm{~g}$ from the Beijing Vital River Laboratory Animal Technology Co., Ltd. (Beijing, China) (qualification certificate No.: 11400700010718; laboratory animal application license No.: 
SCXK [J.] 2012-0001). The rats were fed with dedicated feed (Beijing Vital River Laboratory Animal Technology Co., Ltd.) in a sterilized moist cage with a barrier system.

\section{Methods}

\section{Construction and preparation of the adenovirus NSF small hairpin RNA (Adv-NSF-shRNA) vector}

The NSF gene sequence was selected from GenBank (accession No. NM_021748.1). Using the software siRNA Target Designer version 1.51, interfering oligonucleotide sequences targeting the N-terminal region of the NSF gene were designed, along with the positive-sense and antisense strands of the siRNA. The sequences were connected with a looped oligonucleotide (10 nt) to constitute a short hairpin RNA (shRNA).

It is thought that the synthesis of two single strands of a DNA template encoding a shRNA might help achieve a DNA double-strand template of shRNA by single-strand annealing. An RNA III polymerase transcription termination site was connected to the template strand. MluI and HindIII enzyme cleavage sites were designed into the two termini, which were utilized to clone the shRNA molecule between the MluI and HindIII restriction sites of the multi-cloning site on the siRNA adenovirus pRNAT-H1.1/Adeno (SD1219) vector.

Following double digestion of the SD1219 vector with MluI and HindIII, the linear vector was obtained by $1 \%$ agarose gel electrophoresis. The linear vector was then connected to the $N S F$-shRNA DNA template via the corresponding restriction sites on both termini via T4 ligase. DH5 $\alpha$ competent bacteria were transfected by the ligated product, and inoculated into Luria-Bertani-kanamycin culture medium at $37^{\circ} \mathrm{C}$ and cultivated overnight. Bacteria were isolated, and plasmid DNA was extracted using a plasmid extraction kit (Shanghai Shinegene Company, Shanghai, China) and labeled for cryopreservation after digestion and sequencing identification with $M l u \mathrm{I}$ and HindIII. The purification and identification of Adv-NSF-shRNA were achieved as previously described (Ming, 2011). Viral titering was conducted using the $50 \%$ tissue culture infective dose (TCID50) method.

\section{MI modeling}

The MI model was established by ligating the anterior descending branch of the coronary artery in rats, as previously reported (Chen et al., 2010). For this study, thirty-six SpragueDawley rats were weighed, sorted according to their weight, and randomly divided into the experimental group (injected with recombinant adenovirus), the control group (injected with negative recombinant adenovirus), and the normal saline group ( $\mathrm{N}=12$ in each group). Each group was caged together.

A concentration of $0.4 \mathrm{~mL} / 100 \mathrm{~g} 1 \%$ pentobarbital sodium was applied for abdominal anesthesia, and the BL-420 Biological Functional Experimental System (Beijing Loled Science Traffic Co. Ltd., Beijing, China) was attached onto the subject animal to record the electrocardiogram. Using a tracheal cannula, a thoracotomy was conducted between the fourth and fifth left ribs. The HX-300S respirator (Chengdu Technology \& Market Co., Ltd., Chengdu, China) was then connected, with a breathing frequency of 80 times/min, a $2: 1$ respiratory quotient, and $7-10 \mathrm{~mL}$ tidal volume which was adjusted according to the 
inflation of the lungs. The anterior descending branch of the left coronary artery was tied with 8-0 noninvasive silk thread by inserting a needle to a depth of $0.2-0.4 \mathrm{~mm}$. After the branch had been tied off, the antetheca and apex of the left ventricle turned pale. By electrocardiographic monitoring, observation of ST segment elevation in the V1 lead suggested that successful ligation had occurred.

After successful modeling, the experimental group was injected with $1.2 \times 10^{9} \mathrm{TU} /$ $\mathrm{mL} N S F$-siRNA adenovirus at the top, bottom, and right and left sides of the left ventricular free wall, with $50 \mu \mathrm{L}$ injected at each point for a $200-\mu \mathrm{L}$ total injection volume. The negative control and normal saline groups were administered the same amount of negative control adenovirus and $0.9 \% \mathrm{NaCl}$, respectively. After dosing, the chest was sewn with 0 silk thread sutures, and residual blood was extracted. Negative pressure was maintained in the chest as the breathing machine was removed, and the muscle and skin were stitched. The tracheal cannula was reserved for preventing blockage of the airway after surgery. Furthermore, warm light and a hot-water bottle were used to maintain the body temperature of the rats. After revival from anesthesia, a small amount of clean water was provided to the rats, and the tracheal cannula was removed. Rats in the same experimental group were returned to their shared cage, and observed for $4 \mathrm{~h}$ after surgery. Penicillin was injected into the muscle 5 days after surgery to prevent infection.

\section{Measurement of the left ventricular ejection fraction (LVEF) with ultrasonic cardiogram}

The rats were weighed again after 2 weeks, $0.4 \mathrm{~mL} / 100 \mathrm{~g} \mathrm{1 \%}$ pentobarbital sodium was applied for abdominal anesthesia, and the electrocardiogram was recorded. After anesthesia, the LVEF values of the rats were measured with an ultrasonic cardiogram.

\section{Measurement of left ventricular end-diastolic pressure (LVEDP) and the maximum rate of rise in left ventricular pressure $(+d p / d t$ max $)$}

LVEDP and $+\mathrm{dp} / \mathrm{dt}$ max were measured using the BL-420 Biological Functional Experimental System. After anesthesia, the rats were placed on their backs. Tubing was placed into the left ventricular space through the right external carotid artery, and this tubing was filled with $1250 \mathrm{0U} / \mathrm{L}$ heparin sodium normal saline diluent. The tubing was then connected to the Biological Functional Experimental System, and the LVEDP and $+\mathrm{dp} / \mathrm{dt}$ max values of the left ventricles of the rats were measured. A pressure transducer was placed at the same level as the left ventricle, and a zero setting was used before and after measurements to ensure reliability of the data.

\section{TTC staining}

Following determination of cardiac function outcome measurements, the rats were sacrificed and their hearts were dissected and washed repeatedly with ice brine. The connective tissue on the surface of the heart was removed, and then serial sections were cut. The sections were stained with $2 \%$ TTC solution for $20 \mathrm{~min}$ in the dark in a shaking water bath with constant temperature $\left(37^{\circ} \mathrm{C}\right)$. The sections were photographed and the software ImageJ was used to determine the MI area. 


\section{Statistical analysis}

Data are reported as means \pm standard deviation. A homogeneity test of variance was conducted with the SPSS 16.0 statistical software (SPSS, Chicago, IL, USA). If the variances were not even, variable conversion was performed to reach homogeneity. The $t$-test was used for inter-group comparisons and $\mathrm{P}<0.05$ was taken to indicate statistical significance.

\section{RESULTS}

\section{Construction and preparation of Adv-NSF-shRNA}

The oligonucleotide sequence used to target the $\mathrm{N}$-terminal region of the NSF gene was 5'-TAG GAC TGG TCG TTG GAA ACA-3' (21 nt) (Figure 1). The viral titer calculated using TCID50 was $1.2 \times 10^{10} \mathrm{TU} / \mathrm{mL}$.

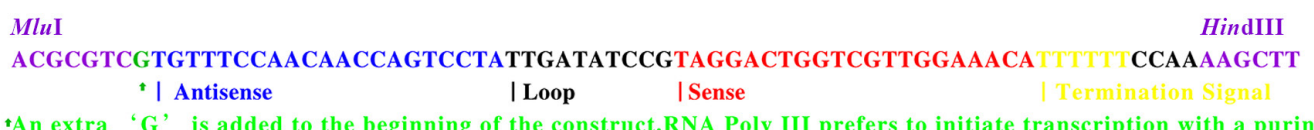

Figure 1. shRNA sequence diagram. An extra "G" (green arrow) was added to the beginning of the construct, as RNA Polymerase III prefers to initiate transcription with a purine.

\section{MI modeling results}

An electrocardiogram was used to monitor the arterial ligation operation. First, the normal V1 lead was recorded (Figure 2A). During the operation, ST segment elevation in the V1 lead indicated successful ligation of the anterior descending branch of the left coronary artery (Figure 2B). Two weeks later, a pathological Q wave was observed in the V1 lead (Figure 2C).

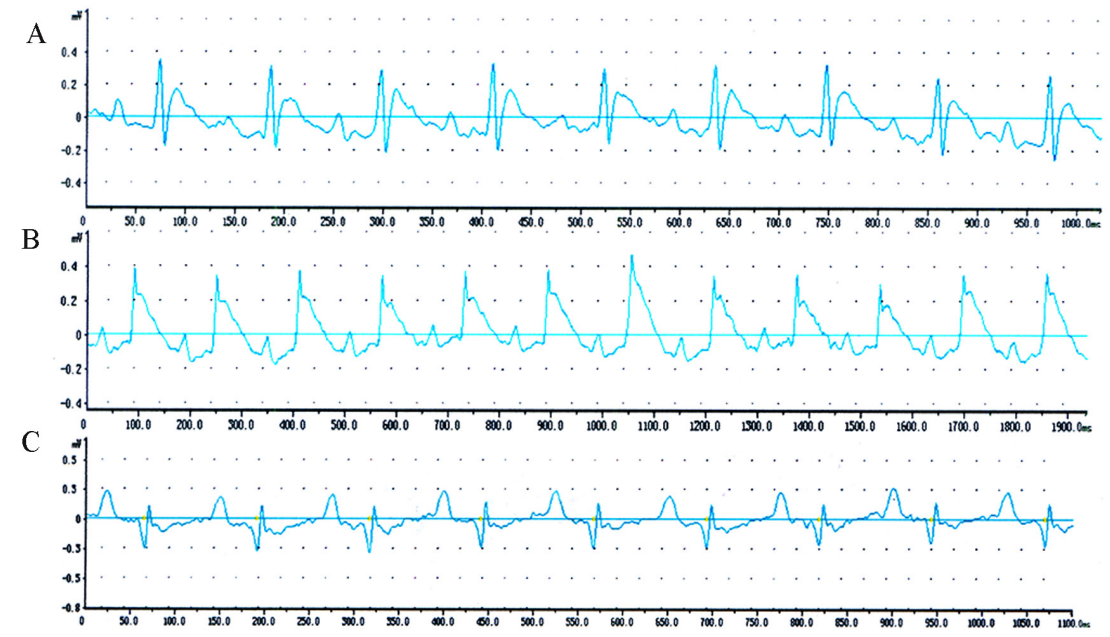

Figure 2. Normal electrocardiogram of lead V1. A. Normal electrocardiogram of lead V1; B. electrocardiogram in lead V1 showing ST segment elevation after ligation of the anterior descending branch of the coronary artery; $\mathbf{C}$. the pathological Q wave in lead V1 as recorded by an electrocardiogram. 
A total of 36 rats with acute MI were prepared for the monitoring experiments. During surgery, six died from arrhythmia, and 3 days after the surgery, eight died from malignant arrhythmia. Only 22 rats survived until the end of the experiment. Of these, 8 rats survived in the normal saline group, and 7 rats survived in each of the control and experimental groups. The survival rate was $61.1 \%$, and none of the rats became infected after the surgery. After the experiment, failure of MI modeling was confirmed in two rats by hematoxylin and eosin (H\&E) staining. Therefore, these animals (1 rat each in the normal saline and the experimental groups) were excluded from the study. Among the rats that survived surgery, the success rate of MI modeling was $90.9 \%$.

\section{LVEF values}

There were significant differences in LVEF among the groups (Table 1).

Table 1. Comparison of LVEF, LVEDP, and $+\mathrm{dp} / \mathrm{dt}$ max in rats among the experimental groups.

\begin{tabular}{lccc}
\hline & Normal saline group & Control group & Experimental group \\
\hline LVEF $(\%)$ & $37.5 \pm 4.5$ & $34.0 \pm 6.0$ & $46.0 \pm 7.5^{\mathrm{a}, \mathrm{b}}$ \\
LVEDP $(\mathrm{mmHg})+\mathrm{dp} / \mathrm{dt}$ max & $26.58 \pm 8.97$ & $29.47 \pm 9.94$ & $18.51 \pm 6.87^{\mathrm{a}, \mathrm{b}}$ \\
(mmHg/s) $10^{3}$ & $5.24 \pm 1.53$ & $4.33 \pm 1.19$ & $9.74 \pm 1.16^{\mathrm{a}, \mathrm{b}}$ \\
MI area (\%) & $36.3 \pm 6.4$ & $38.3 \pm 4.2$ & $34.7 \pm 5.0$ \\
\hline
\end{tabular}

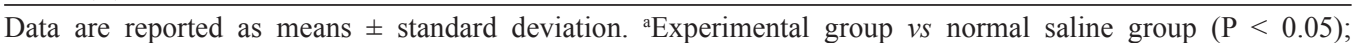
bexperimental group $v s$ control group $(\mathrm{P}<0.05) . \mathrm{LVEF}=$ left ventricular ejection fraction; $\mathrm{LVEDP}=$ left ventricular end-diastolic pressure $;+\mathrm{dp} / \mathrm{dt} \max =$ maximum rate of rise in left ventricular pressure; $\mathrm{MI}=$ myocardial infarction .

\section{LVEDP and +dp/dt max values}

The pressure waveform of the left ventricle is shown in Figure 3. There were significant differences in LVEDP and $+\mathrm{dp} / \mathrm{dt}$ max among the groups (Table 1).

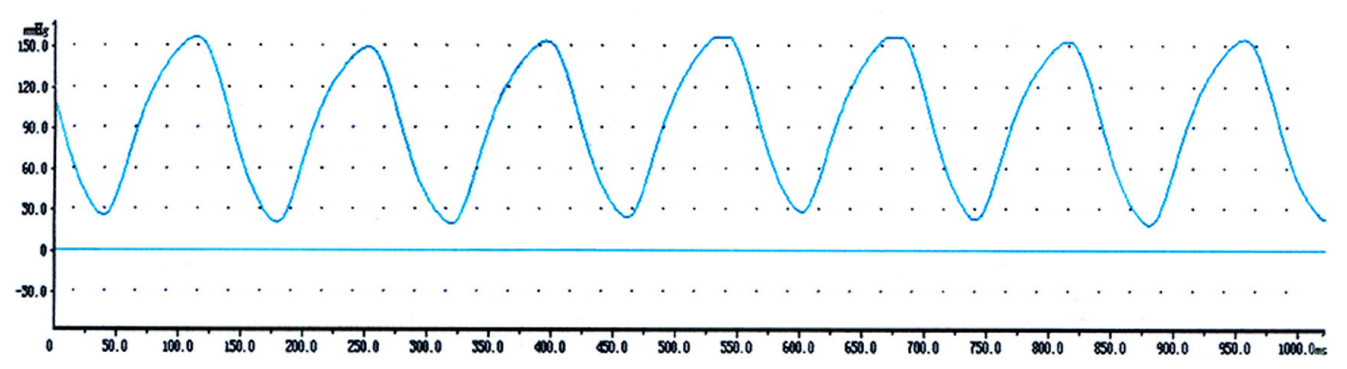

Figure 3. Pressure waveform of the left ventricle as shown by the BL-420 Biological Functional Experimental System (Beijing Loled Science Traffic Co. Ltd.).

\section{TTC staining}

Following TTC staining, the non-infarcted area stained dark red, while the infarcted area became a greyish-white color (Figure 4). There were no distinct statistical differences in the calculated MI area among the three groups (Table 1). 


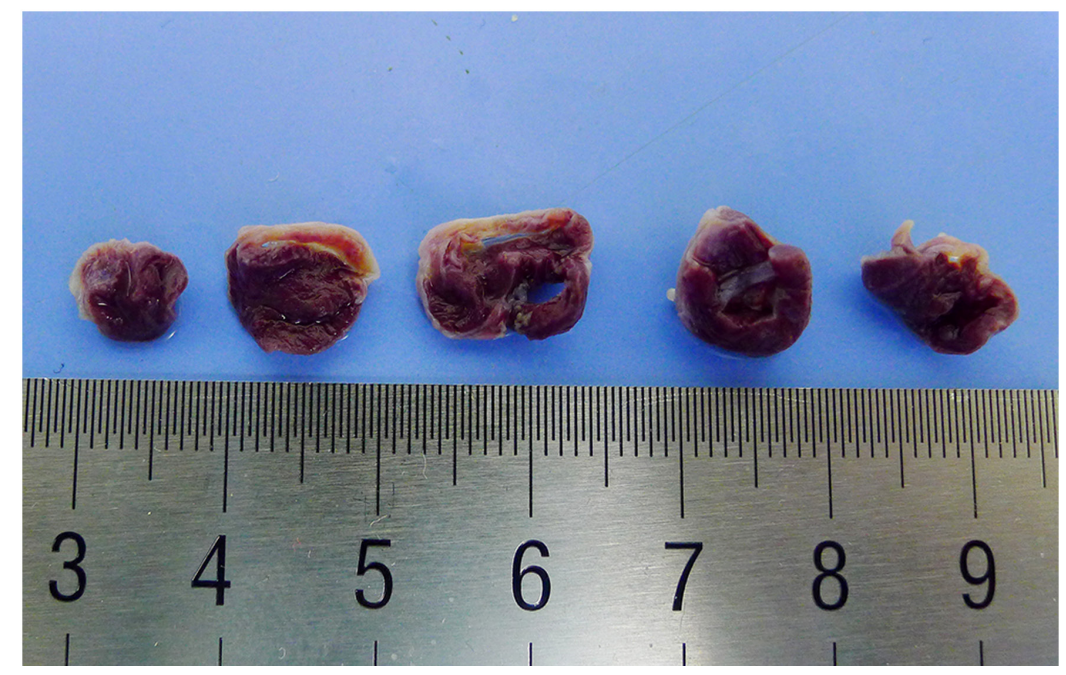

Figure 4. TTC staining of heart sections. TTC $=2,3,5$,-triphenyl tetrazolium chloride.

\section{DISCUSSION}

Currently, the morbidity of acute MI is showing an increasing trend across the world. As chronic heart failure often occurs after MI, the protection of cardiac function after MI has become an important clinical research topic.

As previously mentioned, various studies have shown that NSF plays a vital role in thrombogenesis, inflammatory responses, and vasospasm (Lowenstein et al., 2005; Lowenstein and Tsuda, 2006). The function of NSF and its regulation have been well studied (Matsushita et al., 2005; Cipriano et al., 2013). NSF-siRNA can induce a decrease in NSF mRNA expression and decrease the synthesis of NSF protein, thus restricting the release of WPBs in endothelial cells. RNA interference (RNAi) is a process by which genes are silenced by short oligonucleotides complementary to the target region, most commonly through posttranscriptional interactions with mRNA. RNAi technology is an emerging strategy to block gene production. Experimentally, long double-stranded RNA molecules are split into siRNA oligonucleotides by the endogenous Dicer enzyme after being imported into the cell. These efficiently combine with the target gene mRNA molecules and interfere with protein translation; thus, siRNAs have been used for such diverse functions as curing disease or investigating the function of a target gene. Compared to technologies such as targeted gene knockout or the use of pharmaceutical agents, which often have non-specific effects, RNAi offers many advantages for the research of functional gene groups and related aspects (Matsushita et al., 2005; Cipriano et al., 2013). Based on previous studies, we established MI modeling in rats and injected the recombinant adenovirus $N S F$-siRNA around the infarcted area. Two weeks after MI, we found that the cardiac function indices of the experimental group were significantly superior to those in the control and the normal saline groups. The mechanism involved might be related to the local injection of $N S F$-siRNA after MI, a restriction of $N S F$ target gene expression, a decreased release of WPBs, a decline in thrombus and inflammatory factor levels, weakening of myocardial stunning, or myocardial hibernation effects, as well as to the 
preservation of cardiac function in semi-infarcted areas. The results suggest that RNAi technology has great value in research and for future applications for the prevention and treatment of diseases of the cardiovascular system such as atherosclerosis and acute coronary syndrome.

In this experiment, no distinct statistical differences in the MI area of each group were identified, which might be related to the method used to establish the MI model. Since the ligation of the anterior descending branch is an acute mechanical ligation, the effective collateral circulation cannot be established within a short period, which might result in the treatment being insufficient to decrease the MI area.

In conclusion, our study demonstrated that adenovirus vector-mediated RNA interference effectively improved cardiac function in rats following MI. This result might contribute to the development of a micro-molecule RNA drug. RNAi might also constitute a new approach for treating cardiovascular disease in the future. However, some of the components of recombinant vectors might give rise to host immunoreaction; in addition, various other factors need to be determined prior to clinical use of this technology such as the time of active duration, ideal selection of a vacant vector, selection of helper virus qualities, viral purification, and biological security. Therefore, RNAi as a clinical treatment measure still has many problems that need to be overcome (Sioud, 2011; Hsu and Yang, 2014). The solution to these problems might be reliant upon further basic and clinical research in the future.

\section{Conflicts of interest}

The authors declare no conflict of interest.

\section{ACKNOWLEDGMENTS}

Research supported by the Beijing Natural Science Foundation (\#7083108). We thank Professor Tiemin Ma for assisting us in establishing the MI model in rats and measuring LVEDP and $+\mathrm{dp} / \mathrm{dt}$ max.

\section{REFERENCES}

Chen BJ, Feng JX, Su XX, Meng LQ, et al. (2010). Effects of Tongguan Capsule on post-myocardial infarction ventricular remodeling and cardiac function in rats. Chin. J. Integr. Med. 16: 157-161.

Cipriano DJ, Jung J, Vivona S, Fenn TD, et al. (2013). Processive ATP-driven substrate disassembly by the N-ethylmaleimide-sensitive factor (NSF) molecular machine. J. Biol. Chem. 288: 23436-23445.

Hsu PY and Yang YW (2014). Gene delivery via the hybrid vector of recombinant adeno-associated virus and polyethylenimine. Eur. J. Pharm. Sci. 52: 62-68.

Lowenstein CJ and Tsuda H (2006). N-ethylmaleimide-sensitive factor: a redox sensor in exocytosis. Biol. Chem. 387: $1377-1383$.

Lowenstein CJ, Morrell CN and Yamakuchi M (2005). Regulation of Weibel-Palade body exocytosis. Trends Cardiovasc. Med. 15: 302-308.

Matsushita K, Morrell CN, Mason RJ, Yamakuchi M, et al. (2005). Hydrogen peroxide regulation of endothelial exocytosis by inhibition of N-ethylmaleimide sensitive factor. J. Cell Biol. 170: 73-79.

Ming X (2011). Cellular delivery of siRNA and antisense oligonucleotides via receptor-mediated endocytosis. Expert Opin. Drug Deliv. 8: 435-449.

Sioud M (2011). Promises and challenges in developing RNAi as a research tool and therapy. Methods Mol. Biol. 703: 173-187.

Yang SX, Yan J, Deshpande SS, Irani K, et al. (2004). Rac1 regulates the release of Weibel-Palade Bodies in human aortic endothelial cells. Chin. Med. J. 117: 1143-1150. 